\title{
Pliability Identification of Elastic Support for Elastic Cantilevered Rod Based on Eigenfrequencies of its Oscillations
}

\author{
Vladimir Zhernakov \\ Department of Strength of Materials \\ Ufa State Aviation Technical University \\ Ufa, Russian Federation \\ sm.ugatu@mail.ru \\ Liliya Nusratullina \\ Department of Mathematics \\ Ufa State Aviation Technical University \\ Ufa, Russian Federation \\ mardliliya@yandex.ru
}

\author{
Victor Pavlov \\ Department of Strength of Materials \\ Ufa State Aviation Technical University \\ Ufa, Russian Federation \\ victor.pavlov.51@yandex.ru
}

\author{
Vilina Kudoyarova \\ Department of Aviation Thermal and \\ Energy Engineering \\ Ufa State Aviation Technical University \\ Ufa, Russian Federation \\ Kudoyarova@inbox.ru
}

\begin{abstract}
Currently, modern turbomachines, for example, aircraft gas turbine engines are equipped with highly intelligent automated monitoring and control systems, including a large number of sensors that control various parameters of the product being operated. In particular, there are sensors that monitor the oscillation frequencies of the most critical structural elements. This paper estimates the ability to determine the support stiffness of a cantilever rod under the two first eigenfrequencies of its oscillations. A similar problem arises in the non-destructive testing of the axial compressor blade root of a gas turbine engine when a certain decision of its further operation possibility or changing its operation mode is can be made on the basis of the blade oscillation eigenfrequencies spectrum. As a result of the implementation of the paper proposed method it was obtained that the identification error does not go beyond $28 \%$, which is quite acceptable for assessing the technical condition of the support in the engine corresponding to the range of existing engines.
\end{abstract}

Keywords-Splines, mathematical modelling, rod vibrations

\section{INTRODUCTION}

The majority of damages occurs in the blades of aircraft gas turbine engines (GTE) are associated with the action of alternating stresses arising from vibrations and are fatigue in nature. The blade oscillations produce the large additional dynamic stresses and cause the material fatigue phenomena. As a result, cracks appear in various places of the blades over time, which is the reason of their destruction. As a rule, the damage of one blade leads to an avalanche-like process of damaging or destroying other blades, disturbing the rotor balance, and other serious engine damage. In order to prevent vibration damages the blade oscillations are investigated in the step of engine design and development.

The blade as any elastic structure has a spectrum of eigenfrequencies and modes of vibration. These indicators are decisive because they fully represent the dynamic properties of the blades, their ability to respond to different types of external influence and determine the blade oscillation. While calculating and studying the eigenfrequency spectra and blade vibration modes, it should be taken into account that unacceptable negative changes may occur in the supports in the operation, that lead to changes in their pliability, which in turn will have a negative effect on the magnitude of eigenfrequency of the GTE compressor blade.

The blade axial compressor is considered in the form of a cantilevered elastic rod with elastic supports at its root when analyzing the oscillations.

Currently, analytical methods have been developed for calculating the natural oscillation frequencies of straight rods with constant cross sections [1-3].

To determine the eigenfrequencies of oscillations for the blade, it is necessary to turn to numerical methods as the blade has a variable cross section. The one of the most effective is the spline method [4-27], the identification of boundary conditions is considered in [28, 29].

In view of the above, it is very important to develop methods for estimation of the mechanical state of certain product structural elements that based on the spectrum of eigenfrequencies for oscillations arising in the structure.

\section{PROBLEM DEFINITION}

Consider a straight rod of length $l$ with a constant cross

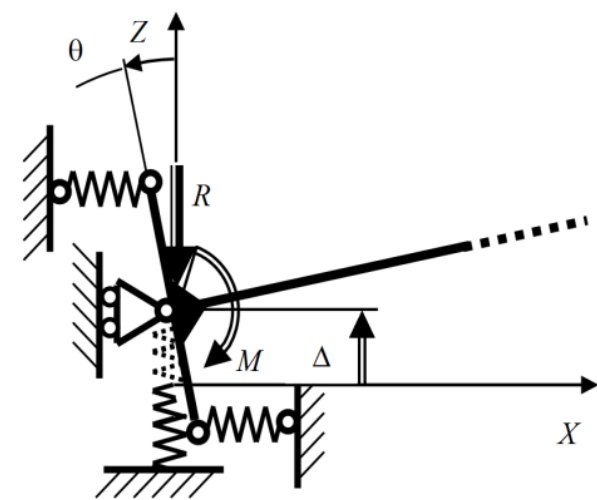

Fig. 1. Elastic cantilevered rod with elastic supports. 
section, defined by the area $A$ and the minimum axial moment of inertia $I_{x}$. The rod made of the material with Young's modulus $E$ and density $\rho$. The rod is elastically fastened at the left end and free at the right end (Fig. 1).

The support of the rod has two types of elastic properties: when the fixing point moves vertically $\Delta$ from the support side a force $R$ is acting on the rod, and when the crosssection of the rod is rotated at an angle from the support side a moment of a couple $M$ is acting on the rod (Fig. 1):

$$
R=\Delta / r, \quad M=\theta / s_{0} .
$$

The coefficients $r, s$ in (1) are the support compliance coefficients. During the working this type structures the unacceptable changes may occur in the support, which will lead to a change in the value of the support compliance coefficients $r, s$. It is very important to be able to timely record the occurrence of this state directly during the product operation.

Based on this, this paper sets the task of developing and putting into practice the method of estimation the support pliability $r, s$ of a cantilevered straight rod by the known first two eigenfrequencies $\omega_{1}, \omega_{2}$ of its vibrations.

\section{EQUATIONS FOR DETERMINING THE EIGENFREQUENCIES OF THE TRANSVERSE OSCILLATIONS OF A ROD}

Consider a straight rod of length $l=0.2 \mathrm{~m}$, having a rectangular cross section of width $b=0.03 \mathrm{~m}$ and height $h=0.01 \mathrm{~m}$. The rod made of the material with Young's modulus $E=2 \cdot 10^{11} \mathrm{~Pa}$ and density $\rho=7.85 \mathrm{~kg} / \mathrm{m}^{3}$. The minimum axial moment of inertia $I_{x}=b h^{3} / 12=2.5 \cdot 10^{-9} \mathrm{~m}^{4}$, the cross-sectional area $A=b h=3 \cdot 10^{-4} \mathrm{~m}^{2}$.

The eigenfrequencies of the rod oscillations are determined [1] - [3] from the equation

$$
E I \frac{\partial^{4} W}{\partial x^{4}}-\mu \omega^{2} W=0, \quad \mu=A \rho,
$$

where $W=W(x)-$ a function describing the form of natural oscillations. With notation:

$$
\begin{cases}x=l \tilde{x}, & x \in[0,1], \\ W=W_{o} \tilde{w}, & \tilde{w} \in[0,1],\end{cases}
$$

where $W_{0}-$ the scale factor with the dimension of a displacement.

Let's introduce the notation for the reduced parameter eigenfrequency $\tilde{\omega}$ of the rod:

$$
\tilde{\omega}^{2}=\frac{\mu l^{4}}{E I} \omega^{2}
$$

then we get the equation (2) in a dimensionless form.

$$
\frac{\partial^{4} \tilde{w}}{\partial \tilde{x}^{4}}-\tilde{\omega}^{2} \tilde{w}=0
$$

The given boundary conditions take the form:

$$
\left\{\begin{array}{llll}
\tilde{w}+\tilde{r} \frac{\partial^{3} \tilde{w}}{\partial \tilde{x}^{3}}=0, & \frac{\partial \tilde{w}}{\partial \tilde{x}}-\tilde{s} \frac{\partial^{2} \tilde{w}}{\partial \tilde{x}^{2}}=0 & \text { при } & \tilde{x}=0, \\
\frac{\partial^{3} \tilde{w}}{\partial \tilde{x}^{3}}=0, & \frac{\partial^{2} \tilde{w}}{\partial \tilde{x}^{2}}=0 & \text { при } & \tilde{x}=1,
\end{array}\right.
$$

where the dimensionless pliability of supports are brought into consideration at $\tilde{x}=0$ :

$$
\tilde{r}=r \frac{E I}{l^{3}}, \quad \tilde{s}=s \frac{E I}{l} .
$$

\section{ALGORITHM FOR DETERMINING SUPPORT PLIABILITY}

The general solution of the reduced equation for oscillations (5) has the form [15] - [18]:

$$
\tilde{w}=\tilde{C}_{1} e^{\tilde{\xi} \tilde{x}}+\tilde{C}_{2} e^{-\tilde{\xi} \tilde{x}}+\tilde{C}_{3} \sin \tilde{\xi} \tilde{x}+\tilde{C}_{4} \cos \tilde{\xi} \tilde{x},
$$

where $\tilde{C}_{1}, \tilde{C}_{2}, \tilde{C}_{3}, \tilde{C}_{4}-$ constants of integration, determined from the given boundary conditions, $\tilde{\xi}$-some unknown magnitude.

Let's write the partial derivatives of the function for the reduced forms of natural oscillations:

$$
\left\{\begin{aligned}
\tilde{w}=\tilde{C}_{1} e^{\tilde{\xi} \tilde{x}}+\tilde{C}_{2} e^{-\tilde{\xi} \tilde{x}}+\tilde{C}_{3} \sin \tilde{\xi} \tilde{x}+\tilde{C}_{4} \cos \tilde{\xi} \tilde{x}, & \frac{\partial \tilde{w}}{\partial \tilde{x}}=\tilde{C}_{1} \tilde{\xi} e^{\tilde{\xi} \tilde{x}}-\tilde{C}_{2} \tilde{\xi} e^{-\tilde{\xi} \tilde{x}}+ \\
& +\tilde{C}_{3} \tilde{\xi} \cos \tilde{\xi} \tilde{x}-\tilde{C}_{4} \tilde{\xi} \sin \tilde{\xi} \tilde{x}, \\
\frac{\partial^{2} \tilde{w}}{\partial \tilde{x}^{2}=} & \tilde{C}_{1} \tilde{\xi}^{2} e^{\tilde{\xi} \tilde{x}}+\tilde{C}_{2} \tilde{\xi}^{2} e^{-\tilde{\xi} \tilde{x}}- \\
& -\tilde{C}_{3} \tilde{\xi}^{2} \sin \tilde{\xi} \tilde{x}-\tilde{C}_{4} \tilde{\xi}^{2} \cos \tilde{\xi} \tilde{x}, \\
\frac{\partial^{3} \tilde{w}}{\partial \tilde{x}^{3}=} & \tilde{C}_{1} \tilde{\xi}^{3} e^{\tilde{\xi} \tilde{x}}-\tilde{C}_{2} \tilde{\xi}^{3} e^{-\tilde{\xi} \tilde{x}}- \\
& -\tilde{C}_{3} \tilde{\xi}^{3} \cos \tilde{\xi} \tilde{x}+\tilde{C}_{4} \tilde{\xi}^{3} \sin \tilde{\xi} \tilde{x}, \\
\frac{\partial^{4} \tilde{w}}{\partial \tilde{x}^{4}=} & \tilde{C}_{1} \tilde{\xi}^{4} e^{\tilde{\xi} \tilde{x}}+\tilde{C}_{2} \tilde{\xi}^{4} e^{-\tilde{\xi} \tilde{x}}+ \\
& +\tilde{C}_{3} \tilde{\xi}^{4} \sin \tilde{\xi} \tilde{x}+\tilde{C}_{4} \tilde{\xi}^{4} \sin \tilde{\xi} \tilde{x} .
\end{aligned}\right.
$$

Substitute (9) into the differential equation (5) and then we get:

$$
\begin{aligned}
& \tilde{\xi}^{4}\left(\tilde{C}_{1} e^{\tilde{\xi} \tilde{x}}+\tilde{C}_{2} e^{-\tilde{\xi} \tilde{x}}+\tilde{C}_{3} \sin \tilde{\xi} \tilde{x}+\tilde{C}_{4} \sin \tilde{\xi} \tilde{x}\right)- \\
& -\tilde{\omega}^{2}\left(\tilde{C}_{1} e^{\tilde{\xi} \tilde{x}}+\tilde{C}_{2} e^{-\tilde{\xi} \tilde{x}}+\tilde{C}_{3} \sin \tilde{\xi} \tilde{x}+\tilde{C}_{4} \cos \tilde{\xi} \tilde{x}\right)=0, \\
& \tilde{x} \in[0,1] .
\end{aligned}
$$


By transforming (10), the formula for calculating the eigenfrequency of oscillation of the reduced parameter rod:

$$
\tilde{\omega}=\tilde{\xi}^{2}
$$

Based on (11) let's write:

$$
\tilde{\xi}=\sqrt{\tilde{\omega}} .
$$

Substituting (9) into (6) we get:

$$
\left\{\begin{array}{l}
\left(\tilde{C}_{1}+\tilde{C}_{2}+\tilde{C}_{4}\right)+\tilde{r} \tilde{\xi}^{3}\left(\tilde{C}_{1}-\tilde{C}_{2}-\tilde{C}_{3}\right)=0, \\
\left(\tilde{C}_{1}-\tilde{C}_{2}+\tilde{C}_{3}\right)+\tilde{s} \tilde{\xi}\left(-\tilde{C}_{1}-\tilde{C}_{2}+\tilde{C}_{4}\right)=0, \\
\tilde{C}_{1} e^{\tilde{\xi}}+\tilde{C}_{2} e^{-\tilde{\xi}}-\tilde{C}_{3} \sin \tilde{\xi}-\tilde{C}_{4} \cos \tilde{\xi}=0, \\
\tilde{C}_{1} e^{\tilde{\xi}}-\tilde{C}_{2} e^{-\tilde{\xi}}-\tilde{C}_{3} \cos \tilde{\xi}+\tilde{C}_{4} \sin \tilde{\xi}=0 .
\end{array}\right.
$$

Let's write (13) in matrix form:

$$
P C=0,
$$

where $\boldsymbol{C}$ - column vector with $4 \times 1$ size:

$$
C=\left(\begin{array}{llll}
C_{1}, & C_{2} & C_{3} & C_{4}
\end{array}\right)^{T},
$$

$\boldsymbol{P}$ - matrix with $4 \times 4$ size:

$$
\boldsymbol{P}=\left(P_{i k}, \quad i=1, \ldots, 4, \quad k=1, \ldots, 4\right),
$$

with components determined on the basis of (13):

$$
\begin{cases}P_{11}=1+\tilde{r} \tilde{\xi}^{3}, & P_{12}=1-\tilde{r} \tilde{\xi}^{3}, \\ P_{13}=-\tilde{r} \tilde{\xi}^{3}, & P_{14}=1, \\ P_{21}=1-\tilde{s} \tilde{\xi}, & P_{22}=-1-\tilde{s} \tilde{\xi}, \\ P_{23}=1, & P_{24}=\tilde{s} \tilde{\xi}, \\ P_{31}=e^{\tilde{\xi}}, & P_{32}=e^{-\tilde{\xi}}, \\ P_{33}=-\sin \tilde{\xi}, & P_{34}=-\cos \tilde{\xi}, \\ P_{41}=e^{\tilde{\xi}}, & P_{42}=-e^{-\tilde{\xi}}, \\ P_{43}=-\cos \tilde{\xi}, & P_{44}=\sin \tilde{\xi} .\end{cases}
$$

Equation (17) has a nonzero solution only when the determinant consisting of the matrix $\boldsymbol{P}$ components is equal to zero:

$$
D=\operatorname{det}\left[P_{i k}\right]=\left|\begin{array}{llll}
P_{11} & P_{12} & P_{13} & P_{14} \\
P_{21} & P_{22} & P_{23} & P_{24} \\
P_{31} & P_{32} & P_{33} & P_{34} \\
P_{41} & P_{42} & P_{43} & P_{44}
\end{array}\right|=0 \text {. }
$$

When substituting (17) into (18) and the subsequent deployment of the determinant we obtain the equation with the notation $x=\hat{r}, y=\tilde{s}$ :

$$
p_{11} x y+p_{10} x+p_{01} y+p_{00}=0,
$$

where $p_{i j}, i, j=0,1-$ are some coefficients determined on the basis of matrix $\boldsymbol{P}$ components.

Equation (19) contains two unknowns. To determine the unknowns it is necessary to construct at least two equations of the form (19).

Let's assume that for the specific rod we know the first two eigenfrequencies $\tilde{\omega}_{1}, \tilde{\omega}_{2}$ of oscillations.

This allows constructing two equations of the form (19) according to the above method.

$$
\left\{\begin{array}{l}
a_{11} x y+a_{10} x+a_{01} y+a_{00}=0 \\
b_{11} x y+b_{10} x+b_{01} y+b_{00}=0
\end{array}\right.
$$

with known coefficients $a_{i j}, i, j=0,1$ and $b_{i j}, i, j=0,1$.

If the first equation is subtracted from the second equation of system (20) then we get:

$$
\left(b_{10}-a_{10}\right) x+\left(b_{01}-a_{01}\right) y+\left(b_{00}-a_{00}\right)=0 .
$$

From (21) we get:

$$
y=k_{0}+k_{1} x,
$$

where

$$
k_{0}=\frac{b_{00}-a_{00}}{a_{01}-b_{01}}, \quad k_{1}=\frac{b_{10}-a_{10}}{a_{01}-b_{01}} .
$$

If we substitute (23) into the first equation of system (20) then we obtain a quadratic equation relatively the coefficient $x$ :

$$
c_{2} x^{2}+c_{1} x+c_{0}=0
$$

where

$$
\left\{\begin{array}{l}
c_{0}=a_{00}+a_{01} k_{0}, \\
c_{1}=a_{10}+a_{01} k_{1}+a_{11} k_{0}, \\
c_{2}=a_{11} k_{1} .
\end{array}\right.
$$

We find two roots by solving equation (24):

$$
x_{1,2}=\frac{-c_{1} \pm \sqrt{c_{1}^{2}-4 c_{0} c_{2}}}{2 c_{2}}
$$

then we define two roots on the base of (23)

$$
y_{1,2}=k_{0}+k_{1} x_{1,2}
$$

As a result, we obtain two pairs of roots and, accordingly, two pairs of possible stiffness imposed towards the rod. 


\section{THE CALCULATION RESULTS OF PLIABILITY SUPPORT FOR THE ROD WITH A CONSTANT CROSS SECTION}

The analysis of the root rigidity for GTE blades when dimensions close to the size of the considered rod shows that in their operational state for the reduced parameter compliance the range is characterized by intervals:

$$
0 \leq \tilde{r} \leq 1 \cdot 10^{-3}, \quad 0 \leq \tilde{s} \leq 1 \cdot 10^{-1} \text {. }
$$

Based on this, the exact values of the reduced parameter compliance $\tilde{r}_{t}, \tilde{s}_{t}$ were given in Table 1 and the first two reduced eigenfrequencies $\tilde{\omega}_{1}, \tilde{\omega}_{2}$ of oscillations were calculated. Further already on $\tilde{\omega}_{1}, \tilde{\omega}_{2}$ the calculated values of pliability $\tilde{r}, \tilde{s}$ were determined, which are summarized in Tab. 1.

TABLE I. VALUES OF THE PEDUCED PARAMETERS OF SUPPORT'S PLIABILITY FOR A ROD WITH CONSTANT CROSS-SECTION

\begin{tabular}{|c|c|c|c|c|}
\hline \multirow{2}{*}{$\begin{array}{c}\text { Number of } \\
\text { Experiment }\end{array}$} & \multicolumn{4}{|c|}{ A Rod with Constant Cross Section } \\
\cline { 2 - 5 } & \multicolumn{2}{|c|}{ Exact Pliability } & \multicolumn{2}{c|}{ Calculated Pliability } \\
\cline { 2 - 5 } & $\tilde{\boldsymbol{r}}_{\boldsymbol{t}}$ & $\tilde{\boldsymbol{s}}_{\boldsymbol{t}}$ & $\tilde{\boldsymbol{r}}$ & $\tilde{\boldsymbol{s}}$ \\
\hline 1 & 0 & 0 & 0 & $9.35 \cdot 10^{-17}$ \\
\hline 2 & $1 \cdot 10^{-3}$ & $1 \cdot 10^{-3}$ & $1.0007 \cdot 10^{-3}$ & $0.9987 \cdot 10^{-3}$ \\
\hline 3 & $1 \cdot 10^{-2}$ & $1 \cdot 10^{-2}$ & $1.02 \cdot 10^{-2}$ & $0.954 \cdot 10^{-2}$ \\
\hline 4 & $1 \cdot 10^{-3}$ & $1 \cdot 10^{-1}$ & $1.14 \cdot 10^{-3}$ & $0.997 \cdot 10^{-1}$ \\
\hline 5 & $1 \cdot 10^{-2}$ & $1 \cdot 10^{-1}$ & $1.23 \cdot 10^{-2}$ & $0.952 \cdot 10^{-1}$ \\
\hline
\end{tabular}

Tab. 1 shows that the calculated values for the pliability support are very close to the exact ones for a rod with a constant cross section.

\section{EXTENSION OF THE METHOD FOR A ROD WITH A VARIABLE CROSS SECTION}

Real blades of GTE compressors have a variable crosssection along the length; therefore, it is necessary to estimate the proposed method possibilities for rods of variable crosssection. In this case, the eigenfrequencies are determined from the equation $[7,17]$ :

$$
E I \frac{\partial^{4} W}{\partial x^{4}}+2 E \frac{\partial I}{\partial x} \frac{\partial^{3} W}{\partial x^{3}}+E \frac{\partial I}{\partial x} \frac{\partial^{2} W}{\partial x^{2}}-\mu \omega^{2} W=0
$$

where $E=$ const, parameters $\mu, I-$ are functions of $x$ coordinate: $\mu=\mu(x), I=I(x)$.

From [7, 17] let's define in exponential form the expressions for a rod's mass per unit length $\mu$ and the axial moment of inertia $I$ of its cross section:

$$
\mu=\mu_{0} e^{\delta \frac{x}{l}}, \quad I=I_{0} e^{\delta \frac{x}{l}}
$$

where $l$ - length of a rod, $\mu_{0}, I_{0}$ and $\delta$ - some constants.

On the base of (30) we define:

$$
\frac{\partial I}{\partial x}=I_{0} \frac{\delta}{l} e^{\delta \frac{x}{l}}, \quad \frac{\partial^{2} I}{\partial x^{2}}=I_{0}\left(\frac{\delta}{l}\right)^{2} e^{\delta \frac{x}{l}} .
$$

Substituting (30) and (31) into (29) we get:

$$
\begin{aligned}
E I_{0} \frac{\partial^{4} W}{\partial x^{4}}+2 E I_{0} \frac{\delta}{l} \frac{\partial^{3} W}{\partial x^{3}}+ & E I_{0} \frac{\delta^{2}}{l^{2}} \frac{\partial^{2} W}{\partial x^{2}}- \\
& -\mu_{0} \omega^{2} W=0 .
\end{aligned}
$$

With notation (3) the equation for oscillation (32) is been leading to a dimensionless form.

$$
\frac{\partial^{4} \tilde{w}}{\partial \tilde{x}^{4}}+2 \delta \frac{\partial^{3} \tilde{w}}{\partial \tilde{x}^{3}}+\delta^{2} \frac{\partial^{2} \tilde{w}}{\partial \tilde{x}^{2}}-\frac{\mu_{0} l^{4}}{E I_{0}} \omega^{2} \tilde{w}=0 .
$$

Let's introduce the designation for the reduced parameter of eigenfrequency of the rod oscillations which is determined by the formula:

$$
\tilde{\omega}^{2}=\frac{\mu_{0} l^{4}}{E I_{0}} \omega^{2} .
$$

Taking into account (34) the equation (33) is reduced to the form

$$
\frac{\partial^{4} \tilde{w}}{\partial \tilde{x}^{4}}+2 \delta \frac{\partial^{3} \tilde{w}}{\partial \tilde{x}^{3}}+\delta^{2} \frac{\partial^{2} \tilde{w}}{\partial \tilde{x}^{2}}-\tilde{\omega}^{2} \tilde{w}=0, \quad \tilde{x} \in[0,1]
$$

With notation

$$
\lambda_{1}=\sqrt{\tilde{\omega}+\frac{\delta^{2}}{4}}, \quad \lambda_{2}=\sqrt{\tilde{\omega}-\frac{\delta^{2}}{4}} .
$$

The exact solution of the equation (35) has the form [17]:

$$
\tilde{w}=e^{-\frac{\delta}{2} \tilde{x}} F(\tilde{x})
$$

where

$$
\begin{aligned}
& F(\tilde{x})=C_{1} e^{\lambda_{1} \tilde{x}}+C_{2} e^{-\lambda_{1} \tilde{x}}+ \\
& +C_{3} \sin \left(\lambda_{2} \tilde{x}\right)+C_{4} \cos \left(\lambda_{2} \tilde{x}\right) .
\end{aligned}
$$

Let's calculate derivatives for function $\tilde{w}=\tilde{w}(x)$ at $\tilde{x}$ up to the third order inclusive on the base of (37) 


$$
\left\{\begin{aligned}
\tilde{w}= & e^{-\frac{\delta}{2} \tilde{x}} F(\tilde{x}), \\
\frac{\partial \tilde{w}}{\partial \tilde{x}}= & -\frac{\delta}{2} e^{-\frac{\delta}{2} \tilde{x}} F(\tilde{x})+e^{-\frac{\delta}{2} \tilde{x}} \frac{\partial F(\tilde{x})}{\partial \tilde{x}}, \\
\frac{\partial^{2} \tilde{w}}{\partial \tilde{x}^{2}}= & \frac{\delta^{2}}{4} e^{-\frac{\delta}{2} \tilde{x}} F(\tilde{x})-\delta e^{-\frac{\delta}{2} \tilde{x}} \frac{\partial F(\tilde{x})}{\partial \tilde{x}}+ \\
& +e^{-\frac{\delta}{2} \tilde{x}} \frac{\partial^{2} F(\tilde{x})}{\partial \tilde{x}^{2}}, \\
\frac{\partial^{3} \tilde{w}}{\partial \tilde{x}^{3}}= & -\frac{\delta^{3}}{8} e^{-\frac{\delta}{2} \tilde{x}} F(\tilde{x})+\frac{3 \delta^{2}}{4} e^{-\frac{\delta}{2} \tilde{x}} \frac{\partial F(\tilde{x})}{\partial \tilde{x}}- \\
& -\frac{3 \delta}{2} e^{-\frac{\delta}{2} \tilde{x}} \frac{\partial^{2} F(\tilde{x})}{\partial \tilde{x}^{2}}+e^{-\frac{\delta}{2} \tilde{x}} \frac{\partial^{3} F(\tilde{x})}{\partial \tilde{x}^{3}},
\end{aligned}\right.
$$

substituting the expressions (39) into (6), we obtain a system of equations for the structure similar to (14) where:

$$
\begin{aligned}
& \left\{\begin{array}{l}
P_{11}=1-\tilde{r} \frac{\delta^{3}}{8}+\tilde{r} \frac{3 \delta^{2}}{4} \lambda_{1}-\tilde{r} \frac{3 \delta}{2} \lambda_{1}^{2}+\tilde{r} \lambda_{1}^{3}, \\
P_{12}=1-\tilde{r} \frac{\delta^{3}}{8}-\tilde{r} \frac{3 \delta^{2}}{4} \lambda_{1}-\tilde{r} \frac{3 \delta}{2} \lambda_{1}^{2}-\tilde{r} \lambda_{1}^{3}, \\
P_{13}=\tilde{r} \frac{3 \delta^{2}}{4} \lambda_{2}-\tilde{r} \lambda_{2}^{3}, \\
P_{14}=1-\tilde{r} \frac{\delta^{3}}{8}+\tilde{r} \frac{3 \delta}{2} \lambda_{2}^{2},
\end{array}\right. \\
& \left\{\begin{array}{l}
P_{21}=-\frac{\delta}{2}+\lambda_{1}-\tilde{s} \frac{\delta^{2}}{4}+\tilde{s} \delta \lambda_{1}-\tilde{s} \lambda_{1}^{2}, \\
P_{23}=-\frac{\delta}{2}-\lambda_{1}+\tilde{s} \frac{\delta^{2}}{4}-\tilde{s} \delta \lambda_{2}, \\
P_{24}=-\frac{\delta}{2}-\tilde{s} \frac{\delta^{2}}{4}+\tilde{s} \lambda_{1} \lambda_{1}^{2},
\end{array}\right.
\end{aligned}
$$

$$
\left\{\begin{array}{l}
P_{31}=\frac{\delta^{2}}{4} e^{\lambda_{1}}-\delta \lambda_{1} e^{\lambda_{1}}+\lambda_{1}^{2} e^{\lambda_{1}}, \\
P_{32}=\frac{\delta^{2}}{4} e^{-\lambda_{1}}+\delta \lambda_{1} e^{-\lambda_{1}}+\lambda_{1}^{2} e^{-\lambda_{1}}, \\
P_{33}=\frac{\delta^{2}}{4} \sin \left(\lambda_{2}\right)-\delta \lambda_{2} \cos \left(\lambda_{2}\right)-\lambda_{2}^{2} \sin \left(\lambda_{2}\right), \\
A P_{44}=\frac{\delta^{2}}{4} \cos \left(\lambda_{2}\right)+\delta \lambda_{2} \sin \left(\lambda_{2}\right)-\lambda_{2}^{2} \cos \left(\lambda_{2}\right),
\end{array}\right.
$$

Then the desired values of support pliability are found by formulas (18) - (27).

\section{THE CALCULATION RESULTS OF PLIABILITY SUPPORT FOR A ROD WITH A VARIABLE CROSS SECTION}

The analysis of the root rigidity for GTE blades when dimensions close to the size of the considered rod shows that in their operational state for the reduced parameter compliance the range is characterized by intervals (28).

The variable section of the rod is setting by different values of the parameter $\delta=-2 ;-1 ; 1 ; 2$.

Bared in mind this, the exact values of the reduced parameter compliance $\tilde{r}_{t}, \tilde{s}_{t}$ were given in Tab. 2, Tab. 3, Tab. 4 and Tab. 5 and the first two reduced eigenfrequencies $\tilde{\omega}_{1}, \tilde{\omega}_{2}$ of oscillations were calculated. Further already on $\tilde{\omega}_{1}, \tilde{\omega}_{2}$ the calculated values of pliability $\tilde{r}, \tilde{s}$ were determined, which are summarized in Tab. 2, Tab. 3, Tab. 4 and Tab. 5.

The calculation results for a rod of variable cross section at $\delta=-1$ are presented in Tab. 2 .

TABLE II. VALUES OF THE PEDUCED PARAMETERS OF SUPPORT'S PLIABILITY FOR A ROD WITH VARIABLE CROSS-SECTION

\begin{tabular}{|c|c|c|c|c|}
\hline \multirow{2}{*}{$\begin{array}{c}\text { Number of } \\
\text { Experiment }\end{array}$} & \multicolumn{4}{|c|}{ A Rod with Variable Cross Section at $\boldsymbol{\delta}=-\mathbf{1}$} \\
\cline { 2 - 5 } & $\tilde{\boldsymbol{r}}_{\boldsymbol{t}}$ & $\tilde{\boldsymbol{s}}_{\boldsymbol{t}}$ & $\tilde{\boldsymbol{r}}$ & $\tilde{\boldsymbol{s}}$ \\
\hline 1 & 0 & 0 & $-3.60 \cdot 10^{-16}$ & $4.18 \cdot 10^{-16}$ \\
\hline 2 & $1 \cdot 10^{-3}$ & $1 \cdot 10^{-3}$ & $0.999 \cdot 10^{-3}$ & $1.000 \cdot 10^{-3}$ \\
\hline 3 & $1 \cdot 10^{-2}$ & $1 \cdot 10^{-2}$ & $1.02 \cdot 10^{-2}$ & $0.986 \cdot 10^{-2}$ \\
\hline 4 & $1 \cdot 10^{-3}$ & $1 \cdot 10^{-1}$ & $1.08 \cdot 10^{-3}$ & $0.999 \cdot 10^{-1}$ \\
\hline 5 & $1 \cdot 10^{-2}$ & $1 \cdot 10^{-1}$ & $1.19 \cdot 10^{-2}$ & $0.984 \cdot 10^{-1}$ \\
\hline
\end{tabular}

The calculation results for a rod of variable cross section at $\delta=1$ are presented in Tab. 3 . 
TABLE III. VALUES OF THE PEDUCED PARAMETERS OF SUPPORT'S PLIABILITY FOR A ROD WITH VARIABLE CROSS-SECTION

\begin{tabular}{|c|c|c|c|c|}
\hline \multirow{2}{*}{$\begin{array}{c}\text { Number of } \\
\text { Experiment }\end{array}$} & \multicolumn{3}{|c|}{ A Rod with Variable Cross Section at $\boldsymbol{\delta}=\mathbf{1}$} \\
\cline { 2 - 5 } & \multicolumn{2}{|c|}{ Exact Pliability } & \multicolumn{2}{c|}{ Calculated Pliability } \\
\cline { 2 - 5 } & $\tilde{\boldsymbol{r}}_{\boldsymbol{t}}$ & $\tilde{\boldsymbol{s}}_{\boldsymbol{t}}$ & $\tilde{\boldsymbol{r}}$ & $\tilde{\boldsymbol{s}}$ \\
\hline 1 & 0 & 0 & 0 & $7.53 \cdot 10^{-16}$ \\
\hline 2 & $1 \cdot 10^{-3}$ & $1 \cdot 10^{-3}$ & $1.001 \cdot 10^{-3}$ & $0.995 \cdot 10^{-3}$ \\
\hline 3 & $1 \cdot 10^{-2}$ & $1 \cdot 10^{-2}$ & $1.03 \cdot 10^{-2}$ & $0.918 \cdot 10^{-2}$ \\
\hline 4 & $1 \cdot 10^{-3}$ & $1 \cdot 10^{-1}$ & $1.19 \cdot 10^{-3}$ & $0.994 \cdot 10^{-1}$ \\
\hline 5 & $1 \cdot 10^{-2}$ & $1 \cdot 10^{-1}$ & $1.25 \cdot 10^{-2}$ & $0.916 \cdot 10^{-1}$ \\
\hline
\end{tabular}

The calculation results for a rod of variable cross section at $\delta=-2$ are presented in Tab. 4 .

TABLE IV. VALUES OF THE PEDUCED PARAMETERS OF SUPPORT'S PLIABILITY FOR A ROD WITH VARIABLE CROSS-SECTION

\begin{tabular}{|c|c|c|c|c|}
\hline \multirow{2}{*}{$\begin{array}{c}\text { Number of } \\
\text { Experiment }\end{array}$} & \multicolumn{3}{|c|}{ A Rod with Variable Cross Section at $\boldsymbol{\delta}=\mathbf{- 2}$} \\
\cline { 2 - 5 } & \multicolumn{2}{|c|}{ Exact Pliability } & \multicolumn{2}{c|}{ Calculated Pliability } \\
\cline { 2 - 5 } & $\tilde{\boldsymbol{r}}_{\boldsymbol{t}}$ & $\tilde{\boldsymbol{s}}_{\boldsymbol{t}}$ & $\tilde{\boldsymbol{r}}$ & $\tilde{\boldsymbol{s}}$ \\
\hline 1 & 0 & 0 & $-6.55 \cdot 10^{-16}$ & $2.54 \cdot 10^{-16}$ \\
\hline 2 & $1 \cdot 10^{-3}$ & $1 \cdot 10^{-3}$ & $0.998 \cdot 10^{-3}$ & $0.998 \cdot 10^{-3}$ \\
\hline 3 & $1 \cdot 10^{-2}$ & $1 \cdot 10^{-2}$ & $1.01 \cdot 10^{-2}$ & $1.01 \cdot 10^{-2}$ \\
\hline 4 & $1 \cdot 10^{-3}$ & $1 \cdot 10^{-1}$ & $0.999 \cdot 10^{-3}$ & $0.999 \cdot 10^{-1}$ \\
\hline 5 & $1 \cdot 10^{-2}$ & $1 \cdot 10^{-1}$ & $1.16 \cdot 10^{-2}$ & $1.01 \cdot 10^{-1}$ \\
\hline
\end{tabular}

The calculation results for a rod of variable cross section at $\delta=2$ are presented in Tab. 5 .

TABLE V. VALUES OF THE PEDUCED PARAMETERS OF SUPPORT'S PLIABILITY FOR A ROD WITH VARIABLE CROSS-SECTION

\begin{tabular}{|c|c|c|c|c|}
\hline \multirow{2}{*}{$\begin{array}{c}\text { Number of } \\
\text { Experiment }\end{array}$} & \multicolumn{3}{|c|}{ A Rod with Variable Cross Section at $\boldsymbol{\delta}=-\mathbf{2}$} \\
\cline { 2 - 5 } & \multicolumn{2}{|c|}{ Exact Pliability } & \multicolumn{2}{c|}{ Calculated Pliability } \\
\cline { 2 - 5 } & $\tilde{\boldsymbol{r}}_{\boldsymbol{t}}$ & $\tilde{\boldsymbol{s}}_{\boldsymbol{t}}$ & $\tilde{\boldsymbol{r}}$ & $\tilde{\boldsymbol{s}}$ \\
\hline 1 & 0 & 0 & $-6.55 \cdot 10^{-17}$ & $2.54 \cdot 10^{-15}$ \\
\hline 2 & $1 \cdot 10^{-3}$ & $1 \cdot 10^{-3}$ & $1.002 \cdot 10^{-3}$ & $0.991 \cdot 10^{-3}$ \\
\hline 3 & $1 \cdot 10^{-2}$ & $1 \cdot 10^{-2}$ & $1.03 \cdot 10^{-3}$ & $0.879 \cdot 10^{-2}$ \\
\hline 4 & $1 \cdot 10^{-3}$ & $1 \cdot 10^{-1}$ & $1.24 \cdot 10^{-3}$ & $0.990 \cdot 10^{-1}$ \\
\hline 5 & $1 \cdot 10^{-2}$ & $1 \cdot 10^{-1}$ & $1.28 \cdot 10^{-2}$ & $0.879 \cdot 10^{-1}$ \\
\hline
\end{tabular}

From Tab. 2, Tab. 3, Tab. 4 and Tab. 5, it can be seen that for a rod with a variable cross section the calculated values of the support's pliability are also very close to the exact.

\section{ANALYSIS OF THE CALCULATION RESULTS}

Comparison $\tilde{r}_{t}, \tilde{s}_{t}$ and $\tilde{r}, \tilde{s}$ in Tab. 1, Tab. 2, Tab. 3, Tab. 4 and Tab. 5 shows the values $\tilde{r}_{t}, \tilde{s}_{t}$ and $\tilde{r}, \tilde{s}$ practically do not differ for absolutely rigid support at $\widetilde{r}=0_{t}, \tilde{s}_{t}=0$.
Increasing support pliability, the discrepancies increase, but even with the maximum allowable values of pliability from the interval (28) when $\tilde{r}_{t}=1 \cdot 10^{-3}, \tilde{s}_{t}=1 \cdot 10^{-1}$ we have similar values $\tilde{r}=1,28 \cdot 10^{-3}, \tilde{s}=0,870 \cdot 10^{-1}$ that differ by no more than $28 \%$.

\section{CONCLUSION}

This paper has developed the method for identifying the compliance coefficients of the elastic support for a cantilevered rod with constant and variable cross-section based on the first two eigenfrequencies of its oscillations.

The mathematical experiment was realized for estimation of the abilities of a cantilevered rod support, which is similar in properties to GTE blades of a real axial compressor.

As a result of the implementation of the paper proposed method it was obtained that the identification error does not go beyond $28 \%$, which is quite acceptable for assessing the technical condition of the support in the engine corresponding to the range of existing engines.

\section{REFERENCES}

[1] I. M. Babakov, Teoriya Kolebanij (Oscillation Theory). Moscow: Nauka, 1965. (rus).

[2] I. A. Birger, R. R. Mavlyutov, Soprotivlenie Materialov (Strength of Materials). Moscow: Nauka, 1986. (rus)

[3] V. I. Feodos'ev, Soprotivlenie Materialov (Strength of Materials). M.: Nauka, 1972. (rus).

[4] Kudoyarova V., Pavlov V., "Refining of numerical solution for nonlinear transient heat conduction in a plate made of polymer composite material", International Journal of Applied Engineering Research, vol. 10, №18, pp. 39466-39470, 2015.

[5] Nedelcu M., Groza P., Cucu Hortensiu L., "The estimation of dynamic properties of a fixed beam using experimental modal testing", Acta technica napocensis. Series: Applied Mathematics and Mechanics, vol. 55, №3, pp. 559-564, 2012.

[6] Aminbaghai M., Dorn M., Eberhardsteiner J., Pichler B., "A matrixvector operation-based numerical solution method for linear $\mathrm{m}$-th order ordinary differential equations: application to engineering problems", Advances in applied mathematics and mechanics, vol. 5, №3, pp. 269-308, 2013.

[7] Wu Jiong-Yu, "On the limit case of the step-reduction method for calculating non-uniform beam with various sections", Applied mathematics and mechanics - English edition, vol. 11, №8, pp. 795-800, 1990.

[8] Kudoyarova V.M., Pavlov V.P., "The Spline Method for the Solution of the Transient Heat Conduction Problem with Nonlinear Initial and Boundary Conditions for a Plate", Procedia Engineering, vol. 150, pp. 1419-1426, 2016.

[9] Dimarogonas A.D., "Vibration of cracked structures: a state of the art review”, Engineering Fracture Mechanics, vol. 55, № 5, pp. 831-857, 1996.

[10] Freund L.B., Herrmann G., "Dynamic fracture of a beam or plate in plane bending", International journal of applied mechanics, vol. 76, pp.112-116, 1976.

[11] V.S. Zhernakov, V.P. Pavlov, V.M. Kudoyarova, "The Enhanced Spline-Method for Numerical Results of Natural Frequencies of Beams", Procedia Engineering, vol. 176, pp. 438-450, 2017.

[12] Shahin S. Nudehi, "Cantilever Beam Vibration Suppression Using Modal Energy Redistribution", Advances in Acoustics and Vibration, vol. 12, pp. 1-9, 2012.

[13] Mao K., et al., "Simulation and characterization of particledamping in transient vibrations", Journal of Vibration and Acoustics, vol. 126, № 2, pp. 202-211, 2004.

[14] V.S. Zhernakov, V.P. Pavlov, V.M. Kudoyarova, "Spline-Method for Numerical Calculation of Natural-Vibration Frequency of Beam with 
Variable Cross-Section", Procedia Engineering, vol. 206, pp. 710715, 2017.

[15] Movahhedy M., Ismail F., "Reconstruction of mass-spring system from spectral data", Inverse Problems, vol. 1, №4, pp. 315-327, 1995.

[16] Ramesh M.N.V., N. Mohan Rao, "Free Vibration Analysis of Rotating Functionally-Graded Cantilever Beams", International Journal of Acoustics and Vibration, vol. 19, №1, pp. 31-41, 2014.

[17] V.P. Pavlov, V.M. Kudoyarova, "Spline Based Numerical Method for Heat Conduction Nonlinear Problems Solution", Procedia Engineering, vol. 206, pp. 704-709, 2017.

[18] Song X., Zhai H., Liang D. J., "Dynamic Load Identification and Displacement Prediction Based on FBG for a Cantilever Beam", vol. 2, pp. 1-7, 2019.

[19] Vyasarayani C., Sandhu S., McPhee J., "Nonsmooth Modeling of Vibro-Impacting Euler-Bernoulli Beam", Advances in Acoustics and Vibration, vol.12, pp. 1-9, 2012.

[20] V.S. Zhernakov, V.P. Pavlov, V.M. Kudoyarova, "Deformation of Thin Elastic Rod Under Large Deflections", Lecture Notes in Mechanical Engineering, pp. 185-196, 2018.

[21] Cheng Keng-tung, Ding Hua, "On dynamic optimization of Timoshenko beam", Applied mathematics and mechanics - English edition, vol. 4, №1, pp. 69-77, 1983.
[22] Schmieg H., Vielsack P., "Forced vibrations and accumulation of plastic displacements of a compressed beam", Archive of applied mechanics, vol. 55, № 5, pp. 358-367, 1985.

[23] Akhtyamov A.M., Aitbaeva A.A., "Determination of the Type and Parameters of a Beam End Fastening", Azerbaijan Journal of Mathematics, vol. 1, № 2, pp. 79-91, 2011.

[24] Morassi A., Dilena M., "On point mass identification in rods and beams from minimal frequency measurements", Inverse Problems, vol. 10, № 3, pp. 183-201, 2002.

[25] Zahui M., Thomas R., "Beam Vibration Displacement Curve Measurement", International Journal of Acoustics and Vibration, vol. 22, № 1, pp. 111-120, 2017.

[26] Wang D.Q., Wu C.J., Yang R.C., "Free Vibration of the Damping Beam UsingCo-simulation Method Based on the MFT", International Journal of Acoustics and Vibration, vol. 20, № 4, pp. 251-257, 2015.

[27] Raposo C.A., Ferreira J., "Exponential stability for the timoshenko beam by a locally distributed damping", international journal of differential equations, vol. 10, № 4, pp. 75-82, 2005

[28] Akhtyamov A. M, Teoriya Identifikacii Kraevyh Uslovij (Theory of Identification of Boundary Conditions). Ufa: Gilem, 2008. (rus).

[29] Akhtyamov A. M, Teoriya Identifikacii Kraevyh Uslovij i ee Prilozheniya (Theory of Identification of Boundary Conditions and its Applications). Moscow: Fizmatlit, 2009. (rus). 